01.5

\title{
Клонирование химерных состояний в двухслойной сети бистабильных генераторов с запаздывающей обратной связью
}

\author{
(С) Д.Д. Кульминский ${ }^{1,2}$, В.И. Пономаренко ${ }^{1,2}$, М.Д. Прохоров ${ }^{1}$ \\ ${ }^{1}$ Саратовский фрилиал Института радиотехники и электроники им. В.А. Котельникова РАН, \\ Саратов, Россия \\ ${ }^{2}$ Саратовский национальный исследовательский государственный университет им. Н.Г. Чернышевского, \\ Саратов, Россия \\ E-mail: kulminskydd@gmail.com
}

Поступило в Редакцию 7 сентября 2020 г.

В окончательной редакции 6 октября 2020 г.

Принято к публикации 6 октября 2020 г.

Впервые экспериментально исследовано клонирование химерных состояний в двухслойной сети идентичных бистабильных радиотехнических генераторов с запаздывающей обратной связью. Рассмотрены случаи однонаправленной и взаимной связи между слоями сети. Показано, что для клонирования химерных состояний необходимо соответствующим образом выбрать начальные условия генераторов.

Ключевые слова: сети связанных осцилляторов, химерное состояние, генератор с запаздыванием, радиофизический эксперимент.

DOI: 10.21883/PJTF.2021.02.50543.18543

Системы с сетевой структурой чрезвычайно широко распространены в природе, технике и социуме, и их изучение представляет фундаментальный и практический интерес. Особое внимание привлекает задача исследования адаптивных динамических сетей, в которых топология связей между элементами сети может меняться во времени [1]. Показано, что изменение коллективного поведения элементов таких сетей происходит как вследствие внутренней динамики системы [2,3], так и в ответ на внешние воздействия [4]. Важной прикладной задачей для многих приложений является управление коллективной динамикой в сетях, в том числе синхронизация колебательных режимов [1].

В настоящей работе управление коллективной динамикой в адаптивных сетях исследовано на примере химерных состояний, возникающих в ансамблях идентичных осцилляторов и характеризующихся одновременным существованием в ансамбле как осцилляторов, совершающих синхронные колебания, так и осцилляторов, колеблющихся несинхронно. Химерные состояния были обнаружены при теоретических и экспериментальных исследованиях ансамблей связанных осцилляторов [5-7]. На различных модельных системах были обнаружены эффекты синхронизации химерных состояний в модульных сетях [8] и мультиплексных сетях [9-12], в том числе состоящих из бистабильных осцилляторов [13-15]. Эти эффекты типичны для биологических объектов и нейронных сетей мозга $[16,17]$. Недавно $[18,19]$ был обнаружен и изучен новый эффект - клонирование химерных состояний, возникающий при объединении в мультиплексную систему двух кольцевых ансамблей линейно локально связанных бистабильных осцилляторов. Численно было показано, что при определенных зна- чениях силы и времени межкольцевого взаимодействия в кольце с изначально беспорядочным распределением фаз формируется копия химерного состояния, наблюдаемого в другом кольце, с точностью до значений фаз в некогерентной части. В основе эффекта лежит конкурентная динамика пар осцилляторов из разных слоев мультиплексной системы $[18,19]$.

Мы впервые экспериментально исследовали клонирование химерных состояний в двухслойной сети идентичных бистабильных радиотехнических генераторов с запаздывающей обратной связью. Обнаружение нами эффекта клонирования химерных состояний в физическом эксперименте доказывает реальность его существования, а наблюдение эффекта в системах с задержкой, широко распространенных в природе и технике [20], свидетельствует об общности этого нелинейного явления для широкого класса систем.

Рассмотрим сеть, состоящую из двух слоев, каждый из которых представляет собой кольцо из связанных бистабильных генераторов с запаздывающей обратной связью. Динамика такой мультиплексной сети описывается следующими уравнениями:

$$
\begin{gathered}
\varepsilon \dot{x}_{i}^{j}(t)=-x_{i}^{j}(t)+f\left(x_{i}^{j}(t-\tau)\right)+k_{p}\left(x_{i+1}^{j}(t)-x_{i}^{j}(t)\right) \\
+k_{m}(t)\left(x_{i}^{j+1}(t)-x_{i}^{j}(t)\right), \\
k_{m}(t)= \begin{cases}0, & t<t_{1}, \\
k, & t_{1} \leq t<t_{2} \\
0, & t \geq t_{2},\end{cases}
\end{gathered}
$$

где $x(t)$ - напряжение на входе линии задержки генеpaтора, $i=1, \ldots, N-$ номер генератора внутри слоя, $N-$ количество генераторов в слое, $j=1,2-$ номер 

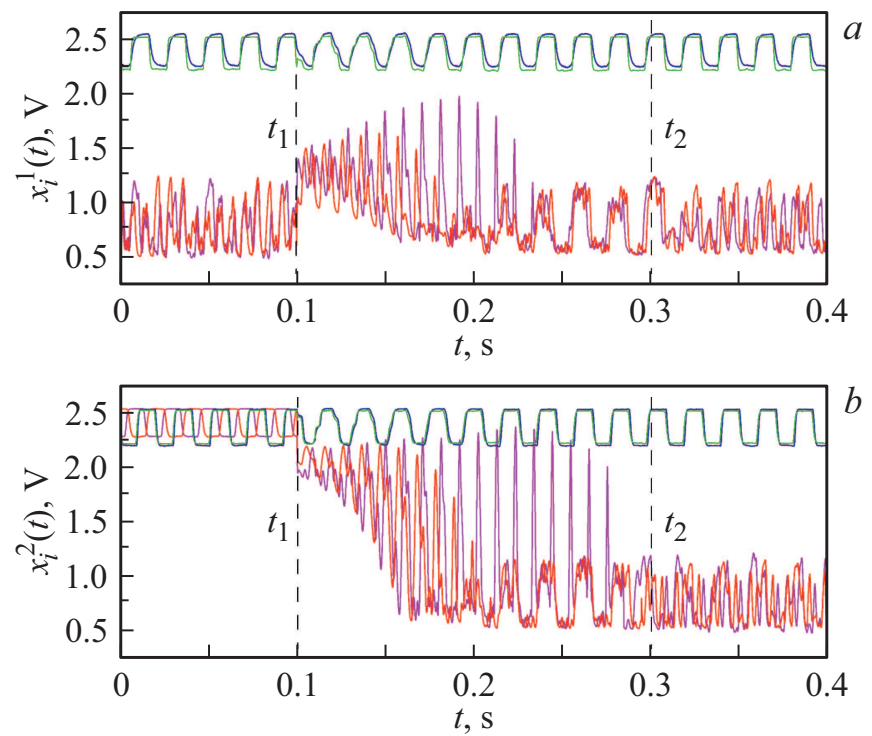

Рис. 1. Экспериментальные временнб́е реализации колебаний напряжения $x_{i}^{j}(t)$ генераторов первого слоя $(a)$ и второго слоя $(b)$ при $\tau=1 \mathrm{~ms}, \varepsilon=0.08 \mathrm{~ms}, k_{p}=0.01$, демонстрирующие клонирование химерного состояния при взаимной связи $k=0.5$ между слоями. Временнь́е реализации $x_{i}^{1}(t)$ и $x_{i}^{2}(t)$ показаны одинаковым цветом (см. электронную версию статьи).

слоя, граничные условия $x_{N+1}^{j}=x_{1}^{j}$ и $x_{i}^{3}=x_{i}^{1}, \varepsilon-$ параметр инерционности, $\tau$ - время запаздывания, $f-$ нелинейная функция, $k_{p}-$ коэффициент связи генераторов внутри слоя, $k_{m}(t)$ - коэффициент связи между слоями сети, параметр $k$ определяет силу связи между слоями, а времена $t_{1}$ и $t_{2}-$ его длительность. В качестве функции $f$ возьмем кубическую функцию

$$
f(x)=a+b(x-d)-c(x-d)^{3},
$$

параметры которой имеют значения $a=1.5, b=2.3$, $c=1.78, d=1.57$. При такой нелинейности в зависимости от начальных условий генератор может совершать два вида колебаний, демонстрируя бистабильность. Один из бистабильных режимов является периодическим с частотой $v_{1} \approx 1 /(2 \tau)$, а другой - хаотическим с основной частотой $\nu_{2} \approx 3 /(2 \tau)$.

Для экспериментальной реализации исследуемой сети радиотехнических генераторов мы использовали предложенный нами недавно подход, в основе которого лежит программный способ формирования сигналов, отвечающих за связь между генераторами [2,3]. Используя такой подход, мы построили экспериментальную установку, которая позволяет задать произвольную архитектуру и тип связей между генераторами и изменять связи в ходе эксперимента.

Рассмотрим случай, когда каждый слой содержит четыре автогенератора с запаздыванием, параметры которых имеют значения $\tau=1 \mathrm{~ms}, \varepsilon=0.08 \mathrm{~ms}, k_{p}=0.01$. Начальные условия выбраны таким образом, что при отсутствии взаимодействия между слоями $(k=0)$ в первом слое реализуется химерное состояние, обладающее следующими свойствами. Генераторы с номерами $i=1,2$ совершают синхронные периодические колебания, а генераторы с номерами $i=3,4$ совершают несинхронные хаотические колебания (рис. 1,a). Начальные условия генераторов второго слоя будем варьировать. Сначала выберем их таким образом, чтобы при $k=0$ все четыре генератора совершали периодические колебания (рис. 1,b).

При включении взаимной связи между слоями в момент времени $t_{1}=0.1 \mathrm{~s}$ генераторы разных слоев начинают взаимодействовать. На рис. 1 приведены временны́ реализации всех динамических переменных $x_{i}^{j}(t)$ при $k=0.5$. Видно, что в результате взаимодействия происходит изменение динамики тех генераторов $\left(x_{3}^{1}(t)\right.$, $x_{4}^{1}(t)$ и $\left.x_{3}^{2}(t), x_{4}^{2}(t)\right)$, колебательные режимы которых качественно различались до включения связи. После окончания переходного процесса, который продолжается некоторое время после момента времени $t_{2}=0.3 \mathrm{~s}$ отключения связи, третий и четвертый генераторы в каждом слое совершают несинхронные хаотические колебания, т.е. во втором слое произошло переключение бистабильных режимов в третьем и четвертом генераторах с низкочастотного периодического на высокочастотный хаотический. По-видимому, это связано с тем, что при заданных параметрах генераторов высокочастотный режим имеет больший бассейн притяжения, чем низкочастотный режим, и побеждает в результате конкуренции. Динамика генераторов $x_{1}^{1}(t), x_{2}^{1}(t)$ и $x_{1}^{2}(t)$, $x_{2}^{2}(t)$, совершающих одинаковые низкочастотные периодические колебаний в обоих слоях, почти не изменилась при включении связи между слоями. Таким образом, в первом слое сохранилось химерное состояние, а во втором слое появилась его копия. Отметим, что при выбранных значениях параметров двухслойной сети (1) клонирование химерного состояния наблюдалось при $\left(t_{2}-t_{1}\right)>0.1 \mathrm{~s}$.

Для наблюдения эффекта клонирования химерного состояния начальные условия генераторов второго слоя должны быть выбраны определенным образом. Если выбрать их так, чтобы все генераторы второго слоя демонстрировали при $k=0$ высокочастотный хаотический режим, то после включения взаимной связи между слоями два периодических генератора первого слоя переходят в хаотический режим, и химерное состояние в первом слое разрушается (рис. 2), причем хаотические колебания генераторов оказываются не синхронизованы между слоями. Аналогичная картина наблюдается в том случае, когда до включения межслойной связи генераторы второго слоя с номерами $i=1,2$ совершают хаотические колебания, а генераторы с номерами $i=3,4$ совершают периодические колебания. В этом случае после включения взаимной связи между слоями все восемь генераторов также оказываются в высокочастотном хаотическом режиме. 

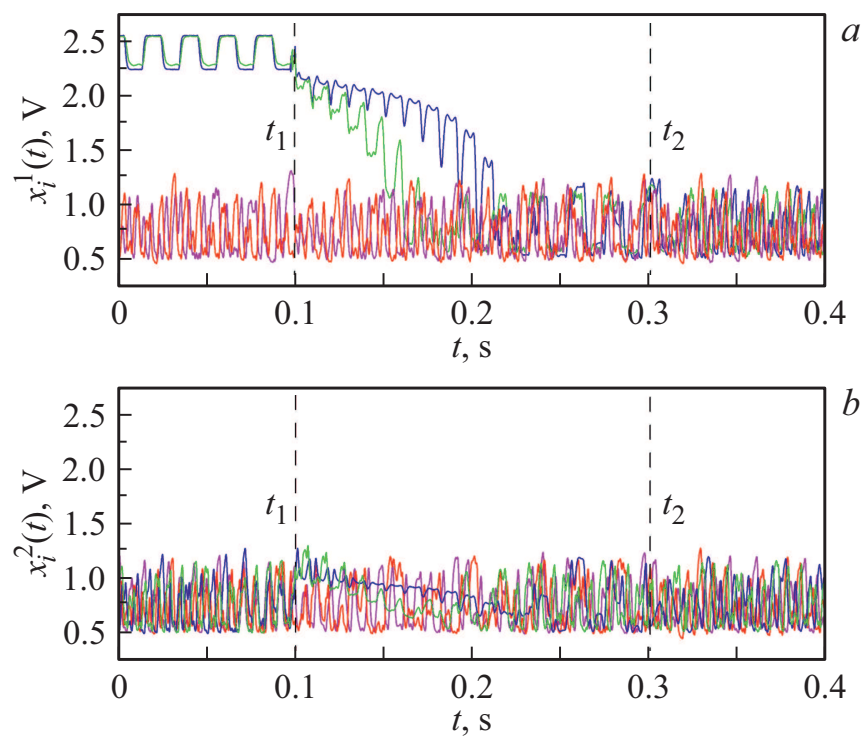

Рис. 2. Экспериментальные временны́е реализации колебаний напряжения $x_{i}^{j}(t)$ генераторов первого слоя $(a)$ и второго слоя $(b)$ при $\tau=1 \mathrm{~ms}, \varepsilon=0.08 \mathrm{~ms}, k_{p}=0.01$, демонстрирующие разрушение химерного состояния при взаимной связи $k=0.5$ между слоями. Временнь́е реализации $x_{i}^{1}(t)$ и $x_{i}^{2}(t)$ показаны одинаковым цветом (см. электронную версию статьи).
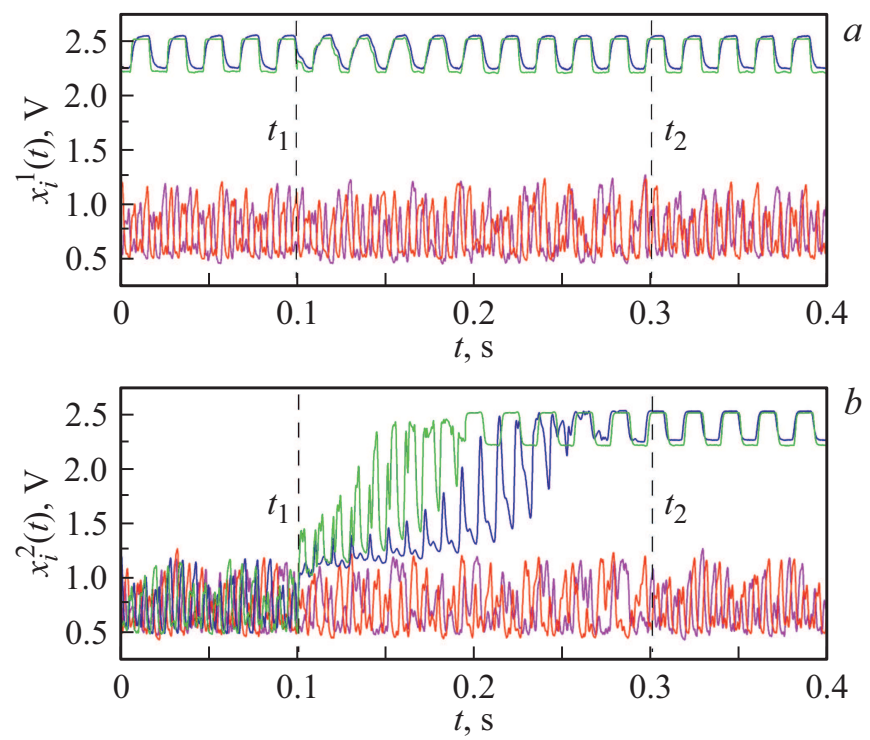

Рис. 3. Экспериментальные временны́е реализации колебаний напряжения $x_{i}^{j}(t)$ генераторов первого слоя $(a)$ и второго слоя (b) при $\tau=1 \mathrm{~ms}, \varepsilon=0.08 \mathrm{~ms}, k_{p}=0.01$, демонстрирующие копирование химерного состояния при однонаправленной связи $k=0.5$ от первого слоя ко второму. Временнб́е реализации $x_{i}^{1}(t)$ и $x_{i}^{2}(t)$ показаны одинаковым цветом (см. электронную версию статьи).

Таким образом, взаимная межслойная связь может привести к изменению бистабильных состояний генераторов как первого, так и второго слоя. Если при включении межслойной связи возникает связь между генераторами, находящимися в одном режиме колебаний, то после выключения связи в обоих генераторах остается этот режим. Если же связь возникает между генераторами, находящимися в разных режимах колебаний, то в результате взаимодействия в каждом из генераторов формируется высокочастотный хаотический режим колебаний.

Рассмотрим теперь случай однонаправленной связи между слоями сети, при котором для второго слоя в уравнении (1) отсутствует последний член, а для первого слоя вместо этого члена появляется слагаемое $k_{m}(t) x_{i}^{2}$. Параметры и начальные условия генераторов первого слоя выберем такими же, как в рассмотренном выше случае, при котором существует химерное состояние. Начальные условия генераторов второго слоя таковы, что все они совершают несинхронные хаотические колебания при $k=0$. После включения однонаправленной межслойной связи $k=0.5$ наблюдается вынужденная синхронизация генераторов второго слоя (рис. 3). В результате во втором слое возникает копия химерного состояния, существующего в первом слое, которая сохраняется и после отключения межслойной связи. В отличие от случая взаимной межслойной связи химерное состояние во втором слое возникает при любых начальных условиях его генераторов. Как видно из рис. 3, при $k_{p} \ll k$ генераторы второго слоя демонстрируют переключение из высокочастотного хаотического режима в низкочастотный периодический, которое не наблюдалось при взаимной связи между слоями.

Итак, нами впервые экспериментально исследовано явление клонирования химерных состояний в двухслойной сети генераторов с запаздывающей обратной связью. Показано, что при взаимной связи между слоями клонирование химерного состояния возникает лишь при определенном выборе начальных условий генераторов и обусловлено конкуренцией колебательных режимов. При сильной однонаправленной связи между слоями копия химерного состояния возникает в результате вынужденной синхронизации и не зависит от начальных условий генераторов.

\section{Благодарности}

Авторы благодарят В.И. Некоркина за полезные обсуждения работы.

\section{Финансирование работы}

Работа выполнена при поддержке гранта Президента РФ, проект МК-1199.2019.8 (дизайн экспериментальной установки), а также Российского фонда фундаментальных исследований, проект № 19-02-00071 (управление коллективной динамикой).

\section{Конфликт интересов}

Авторы заявляют, что у них нет конфликта интересов. 


\section{Список литературы}

[1] О.В. Масленников, В.И. Некоркин, УФН, 187 (7), 745 (2017). DOI: $10.3367 /$ UFNr.2016.10 [Пер. версия: 10.3367/UFNe.2016.10.037902].

[2] В.И. Пономаренко, Д.Д. Кульминский, М.Д. Прохоров, Письма в ЖТФ, 44 (17), 11 (2018).

DOI: $10.21883 /$ PJTF.2018.17.46565.17366 [Пер. версия: 10.1134/S1063785018090109].

[3] Д.Д. Кульминский, В.И. Пономаренко, И.В. Сысоев, М.Д. Прохоров, Письма в ЖТФ, 46 (4), 26 (2020).

DOI: $10.21883 /$ PJTF.2020.04.49046.18018 [Пер. версия: 10.1134/S1063785020020236].

[4] В.И. Пономаренко, Д.Д. Кульминский, А.С. Караваев, М.Д. Прохоров, Письма в ЖТФ, 43 (6), 64 (2017). DOI: 10.21883/PJTF.2017.06.44405.16546 [Пер. версия: 10.1134/S1063785017030208].

[5] D.M. Abrams, S.H. Strogatz, Phys. Rev. Lett., 93, 174102 (2004). DOI: 10.1103/PhysRevLett.93.174102

[6] L.V. Gambuzza, A. Buscarino, S. Chessari, L. Fortuna, R. Meucci, M. Frasca, Phys. Rev. E, 90, 032905 (2014). DOI: 10.1103/PhysRevE.90.032905

[7] V.I. Ponomarenko, D.D. Kulminskiy, M.D. Prokhorov, Phys. Rev. E, 96, 022209 (2017). DOI: 10.1103/PhysRevE.96.022209

[8] J. Hizanidis, N.E. Kouvaris, G. Zamora-López, A. DíazGuilera, C.G. Antonopoulos, Sci. Rep., 6, 19845 (2016). DOI: $10.1038 /$ srep19845

[9] D.V. Kasatkin, V.I. Nekorkin, Chaos, 28, 093115 (2018). DOI: $10.1063 / 1.5031681$

[10] V.A. Maksimenko, V.V. Makarov, B.K. Bera, D. Ghosh, S.K. Dana, M.V. Goremyko, N.S. Frolov, A.A. Koronovskii, A.E. Hramov, Phys. Rev. E, 94, 052205 (2016). DOI: 10.1103/PhysRevE.94.052205

[11] G.I. Strelkova, T.E. Vadivasova, V.S. Anishchenko, Reg. Chaot. Dyn., 23, 948 (2018). DOI: 10.1134/S1560354718070092

[12] Т.Р. Богатенко, А.В. Бух, В.С. Анищенко, Г.И. Стрелкова, Изв. Саратов. ун-та. Нов. сер. Сер. Физика, 20 (1), 42 (2020). DOI: 10.18500/1817-3020-2020-20-1-42-54

[13] Д.С. Щапин, А.С. Дмитричев, В.И. Некоркин, Письма в ЖЭТФ, 106 (9), 591 (2017).

DOI: $10.7868 / \mathrm{S} 0370274 \mathrm{X} 17210135$ [Пер. версия: 10.1134/S0021364017210111].

[14] A.V. Andreev, N.S. Frolov, A.N. Pisarchik, A.E. Hramov, Phys. Rev. E, 100, 022224 (2019) DOI: 10.1103/PhysRevE.100.022224

[15] A.V. Andreev, M.V. Ivanchenko, A.N. Pisarchik, A.E. Hramov, Chaos Solit. Fract., 139, 110061 (2020). DOI: $10.1016 /$ j.chaos.2020.110061

[16] S. Majhi, B.K. Bera, D. Ghosh, M. Perc, Phys. Life Rev., 28, 100 (2019). DOI: 10.1016/j.plrev.2018.09.003

[17] N. Frolov, V. Maksimenko, S. Majhi, S. Rakshit, D. Ghosh, A. Hramov, Chaos, 30, 081102 (2020). DOI: $10.1063 / 5.0019200$

[18] А.С. Дмитричев, Д.С. Щапин, В.И. Некоркин, Письма в ЖЭТФ, $108(8), 574$ (2018). DOI: $10.1134 / \mathrm{S} 0370274 \mathrm{X} 18200109$ [Пер. версия: 10.1134/S0021364018 200079].

[19] A. Dmitrichev, D. Shchapin, V. Nekorkin, Front. Appl. Math. Stat., 5, 9 (2019). DOI: 10.3389/fams.2019.00009

[20] T. Erneux, Applied delay differential equations (SpringerVerlag, N.Y., 2009). DOI: 10.1007/978-0-387-74372-1 